\title{
ARTICULAR CONTACT IN A THREE-DIMENSIONAL MODEL OF THE KNEE
}

\author{
L. BlankeVoort, ${ }^{*}$ J. H. Kuiper, * R. Huiskes* and H. J. Grootengoer $\dagger$ \\ *Biomechanics Section, Institute of Orthopaedies, University of Nijmegen, Nijmegen, The Netherlands \\ and †Department of Applied Mechanics, University of Twente, Enschede, The Netherlands
}

\begin{abstract}
This study is aimed at the analysis of articular contact in a three-dimensional mathematical model of the buman knee-joint. In particular the effect of articular contact on the passive motion characteristics is assessed in relation to experimentally obtained joint kinematics. Two basically different mathematical contact descriptions were compared for this purpose. One description was for rigid contact and one for deformable contact. The description of deformable contact is based on a simplified theory for contact of a thin elastic layer on a rigid foundation. The articular cartilage was described either as a linear elastic material or as a non-linear elastic material. The contact descriptions were introduced in a mathematical model of the knee. The locations of the ligament ineertions and the geometry of the articular surfaces were obtained from a joint specimen of which experimentally determined kinematic data were available, and were used as input for the model. The ligaments were described by non-linear elastic line elements. The mechanical properties of the ligaments and the articular cartilage were derived from literature data. Parametric model evaluations showed that, relative to rigid articular contact, the incorporation of deformable contact did not alter the motion characteristics in a qualitative senee, and that the quantitative changes were small. Variation of the elasticity of the elastic layer revealod that decreasing the surface stifiness caused the ligaments to relax and, as a consequence, increased the joint laxity, particularly for axial rotation. The difference between the linear and the non-linear deformable contact in the knee model was very small for moderate loading conditions. The motion characteristics simulated with the knee model compared very well with the experiments. It is concluded that for simulation of the passive motion characteristics of the knee, the simplified description for contact of a thin linear elastic layer on a rigid foundation is a valid approach when aiming at the study of the motion characteristics for moderate loading conditions. With deformable contact in the knee model, geometric conformity between the surfaces can be modelled as opposed to rigid contact which assumed only point contact.
\end{abstract}

\section{INTRODUCTION}

Recent developments show that three-dimensional mathematical models of the knee-joint develop into powerful tools for functional analysis of the knee, for evaluation of surgical and diagnostic procedures, and for design of artificial joints (e.g. Essinger et al., 1989). A decade ago Wismans (1980) introduced a mathematical model which simulated the quasi-static behaviour of the tibio-femoral joint by calculating the relative equilibrium positions of the tibia and femur for given external loads and kinematic constraints. The ligaments were described by non-linear elastic line elements. The articular surfaces were assumed to be rigid and approximated by surface polynomials of degrees 3 and 4. If the articular surfaces are assumed to be rigid, two problems may occur. If a close match of the anatomic geometry of the joint surfaces is applied through some mathematical function, then the conditions for rigid contact cannot always be satisfied, because of the incompatible local curvatures of the tibial and femoral surfaces at the contact areas. If, on the other hand, the surfaces are approximated by more regular shapes, such as spheres and planes or low degree polynomials, the model characteristics may be less realistic. The purpose of this study was to determine the effects of different mathematical descriptions

Received in final form 4 February 1991. of articular contact and articular surface geometry on the kinematic characteristics of the knee model when compared with experimental passive motion characteristics of the knee. In other words, to what extent can the parameters of the contact and surface descriptions affect the experimental verification of the knee model?

The stiffness models of Crowninshield et al. (1976) and Grood and Hefzy (1982) did not include a description for the articular surfaces, because these models were aimed at the contribution of the ligamentous system to the stiffness characteristics of the knee. Andriacchi et al. (1983) and Essinger et al. (1989) introduced knee models which also took the articular geometry and the ligaments into account. Andriacchi et al. (1983) applied a finite-element-like approach, whereby the individual joint parts were described by elements connected to each other by nodes. They described the ligaments by 21 spring elements, the menisci by two shear beam elements and the articular surfaces by 10 hydrostatic elements which were located at five potential contact regions along each femoral condyle. No parametric analysis was performed with respect to the articular surface description. The model of Essinger et al. (1989) came close to that of Wismans et al. (1980) in its approach, but also included the quadriceps-patellar mechanism and the deformation of the tibial surface. The femoral condyle was assumed to be rigid as to simulate the characteristics of present total knee prostheses. Essinger $e t$ al. 
(1989) restricted their model to the simulation of a particular experimental set-up in which the knee is tested in a simulated standing position under body weight, whereby the flexion angle was controlled by the length of the cable representing the quadriceps muscle (Kurosawa et al., 1985). The solution procedure was based on minimizing the total energy of the system, taking into account the deformation of the tibia by use of a description for linear elastic contact, the ligaments represented by non-linear elastic springs, and a simplified patellar mechanism through which the quadriceps cable and the body weight are acting. A parametric analysis with respect to the deformable contact description in the model was not included in that study.

The three-dimensional mathematical model of the human knee-joint developed in the present study was based on the work of Wismans et al. (1980). The approach, with regard to the mathematical description of deformable contact is similar to that of Essinger et al. (1989) and An et al. (1990), but the underlying theory is more general and can be incorporated in other joint models as well. The geometric data were measured on a knee specimen of which the passive motion characteristics were determined experimentally. The motion patterns, as simulated with the model and the parametric analysis by using different contact descriptions, were evaluated relative to the experimental data. The parameter variations included rigid vs deformable contact and, in the case of deformable contact, variation of the surface stiffness and variation of the polynomial surface approximations.

\section{MITHODS}

\section{General description of the knee model}

The basic assumptions and simplifications in the process of modelling the human knee are similar to those employed by Wismans et al.(1980). The model is aimed at describing the quasi-static behaviour of the tibio-femoral joint for moderate loading conditions. The geometry of the tibial and femoral articular surfaces and the insertion locations of the ligaments are based on geometry measurements (Huiskes et al., 1985; Meijer et al., 1989; Blankevoort et al., 1991) on a knee specimen of which a set of experimental kinematic data was available (Blankevoort et al., 1988). Although the menisci do have a certain role in determining the laxity characteristics of the knee (Bargar et al., 1980; Markolf et al., 1981; Blankevoort et al., 1984), they are not included because of the complexity of incorporating them in the present mathematical formulation of the knee model. The difierences between the present knee model and that of Wismans et al. (1980) include the possibility to constrain each of the three translations and three rotations, and the incorporation of deformable articular contact. Friction between the articular layer is assumed to be negligible. The model describes the position of the femur relative to the tibia for a given configuration of external loads and kinematic constraints. A series of joint positions simulates joint motion.

The position of the femur relative to the tibia was found by solving the equilibrium equations for forces and moments acting on the femur combined with the equations for the contact conditions. The forces and moments were caused by externally applied loads, contact forces, ligament forces and constraint loads, and were functions of the kinematic parameters. The solution was obtained through a Newton-Raphson procedure, in which the analytical partial derivatives of the equations to the kinematic parameters were used.

\section{Relative joint position and kinematic constraints}

The tibia is assumed to be fixed, relative to which the position of the femur is described. Two Cartesian coordinate systems are introduced, a space-fixed system of the tibia and a body-fixed system of the femur (Fig. 1). The $x_{1}$-axis points anteriorly, the $x_{2}$-axis points medially and the $x_{3}$-axis points proximally. Each position of the femur is then characterized by a translation of the origin and three rotations about the axes of the coordinate system. Introducing the translation vector a from $O$ (the origin of the tibial coordinate system) to $\mathbf{O}$ (the origin of the femoral coordinate system) and the rotation matrix $\mathbf{R}$, the position of $a$ material point $\mathbf{P}$ on the femur can be described by the vector $p$ from $O$ to $P$ and the vector $R \cdot P$ from $O$ to $P$. They are related by

$$
\mathbf{p}=\mathbf{a}+\mathbf{R} \cdot \mathbf{p} .
$$

At the reference position where the coordinate systems coincide, $\mathbf{a}$ is a zero vector, $\mathbf{R}$ is the identity matrix and $p$ is the position vector of the material point. The

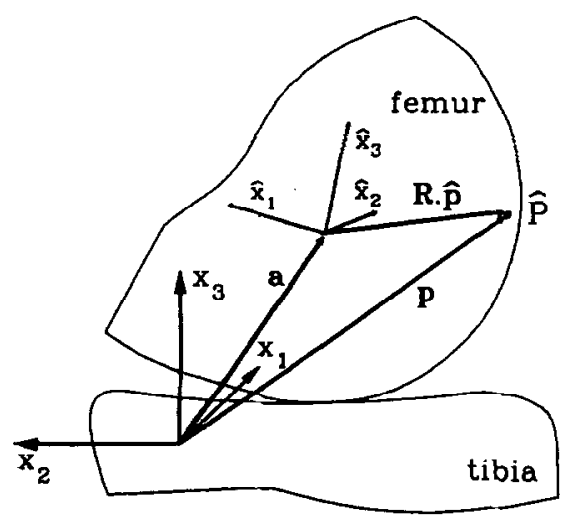

Fig. 1. Schematic representation of the coordinate systems. $\left(x_{1}, x_{2}, x_{3}\right)$ is associated with the tibia and is assumed to be space-fixed; $\left(\hat{x}_{1}, \hat{x}_{2}, \hat{x}_{3}\right)$ is associated to the femur and is assumed to be body-fixed. $A$ material point $\hat{P}$ is described by the vector $p$ relative to the space-fixed system and by the vector $\mathbf{R} \cdot \mathbf{p}$ relative to the body-fixed system. The vector a describes the translation of the body-fixed origin relative to the space-fixed origin. 
reference position is defined for the knee-joint in extension. In this reference position, the origins of the tibial and the femoral coordinate systems are located $15 \mathrm{~mm}$ proximal relative to the insertion of the posterior bundle of the anterior cruciate ligament (pAC) on the tibia, in the approximate region where the helical axes for flexion motions are located (Blankevoort et al., 1988, 1990).

For describing the rotations, the convention of the Joint Coordinate System as proposed by Grood and Suntay (1983) is used in an adapted form. This rotation convention defines the rotations around the body-fixed axes of the moving tibia relative to the fixed femur in the sequence of fiexion, varus and internal rotation. Since the femur is moving relative to the tibia in the present model, a compatible rotation convention is then given by rotations around the body-fixed axes of the femur, provided that the rotations and the rotation sequence are reversed: external rotation ( $x_{3}$-axis), valgus ( $x_{1}$-axis) and extension $\left(x_{2}\right.$ axis) (Woltring, 1991).

One or more of the position parameters may be prescribed and their values can be substituted in the force, moment and contact equations. In order to maintain the applied kinematic constraints, constraint forces and moments have to be introduced:

$$
\begin{aligned}
& \mathbf{f}_{r}=\sum_{1}^{m}-\sigma_{i} a_{i}, \\
& m_{r}=\sum_{1}^{m}-\sigma_{i} \beta_{i},
\end{aligned}
$$

in which $\mathbf{f}$, is the constraint force acting on the femur, $\mathrm{m}_{\mathrm{r}}$ is the constraint moment around $\mathrm{O}, \sigma_{i}$ denotes the magnitude of the constraint load, and $m$ is the number of kinematic constraints. The vectors $\boldsymbol{\alpha}_{i}$ and $\boldsymbol{\beta}_{i}$ are determined by the derivatives of the translation vector and the rotation matrix with respect to the translation and the rotation components, respectively (Appendix A). The values for $\sigma_{l}$ follow from the solution of the system of equilibrium equations and contact equations.

\section{Ligaments}

The ligaments are described by two or more line elements representing different fiber bundles in the ligament. The ligament bundles are assumed to be elastic, which means that the force a ligament exerts on the femur is only a function of its length. Let $\mathbf{s}$ and $\mathbf{R} \cdot \mathbf{8}$ denote the position vectors pointing to the ligament insertions at tibia and femur, respectively. Using equation (1), the unit vector pointing along the force's line of action is given by

$$
v=(a+R \cdot s-s) /\|a+R \cdot s-s\| .
$$

The ligament force on the femur $f_{j}$ and the ligament moment $m_{j}$ abbut $O$ can be expressed by

$$
\begin{aligned}
& \mathbf{f}_{j}=-f_{j}\left(L_{j}\right) \mathbf{v}_{j}, \\
& \mathbf{m}_{j}=\left(\mathbf{R} \cdot \mathbf{s}_{j}\right) \times \mathbf{f}_{j} .
\end{aligned}
$$

A positive force denotes tension in the ligament. If $l$ is the number of ligament bundles modelled, the total ligament force $f_{l}$ and ligament moment $m_{l}$ are found by summation over all ligaments, yielding

$$
\begin{aligned}
\mathbf{f}_{i} & =\sum_{1}^{l}-f_{j}\left(L_{j}\right) \mathbf{v}_{j}, \\
\mathbf{m}_{l} & =\sum_{1}^{l}\left(\mathbf{R} \cdot \mathbf{s}_{j}\right) \times \mathbf{f}_{j} .
\end{aligned}
$$

The function $f_{j}\left(L_{j}\right)$ is assumed to be non-linear for low strains and linear for strains higher than a certain level, which is indicated by $2 \varepsilon_{l}$ (Wismans, 1980):

$$
\begin{array}{ll}
f=\frac{1}{4} k \varepsilon^{2} / \varepsilon_{l}, & 0 \leqslant \varepsilon \leqslant 2 \varepsilon_{l}, \\
f=k\left(\varepsilon-\varepsilon_{l}\right), & \varepsilon>2 \varepsilon_{l}, \\
f=0, & \varepsilon<0,
\end{array}
$$

in which $f$ is the tensile force in a line element, $k$ is the ligament stiffiness and $\varepsilon$ is the strain in the ligament calculated from its length $L$ and the zero-load length $L_{0}$ :

$$
\varepsilon=\left(L-L_{0}\right) / L_{0} .
$$

At the reference (extension) position of the joint, the initial strain of each line element is given by the parameter $\varepsilon_{r}$, which is called the reference strain. This parameter determines the zero load length $L_{0}$, together with the initial length of the ligament, the reference length $L_{r}$ :

$$
\varepsilon_{\mathrm{r}}=\left(L_{\mathrm{r}}-L_{0}\right) / L_{0},
$$

hence

$$
L_{0}=L_{\mathrm{r}} /\left(\varepsilon_{\mathrm{r}}+1\right) .
$$

The different surface approximations in the model will result in slightly different reference positions. Therefore, slightly different reference lengths of the ligament line elements are taken for each surface approximation. These reference lengths are obtained by prescribing the flexion angle, axial rotation, AP translation and, in the case of the deformable surfaces, medial-lateral translation, all set to zero. The resulting line element lengths are considered then to be the reference lengths. In all models (with different surface approximations) the same ligament stiffness and reference strains are chosen (Table 1). They are derived from the literature data Butler et al. (1986) and Danylchuk (1975) (Appendix B). The non-linear strain level parameter $\varepsilon_{l}$ is assumed to be 0.03 for all ligameats (Butler et al., 1986). No quantitative data are available of the reference strains in the ligaments. Therefore, the reference strains of the line elements in the knee model are adapted in such a manner that the motion characteristics of the model approximate the data of previous experiments on the same knee specimen of which the geometry data were measured (Blankevoort et al., 1988). For this purpose, two flexion motions are chosen from the previous experiments, one with an internal torque of $3 \mathrm{Nm}$ and one with an external torque of $3 \mathrm{Nm}$. These are 
Table 1. The stiffiness parameters and reference strains of the ligament line elements

\begin{tabular}{lccc}
\hline Ligament & $\begin{array}{c}\text { Ligament } \\
\text { bundle }\end{array}$ & $\begin{array}{c}k \\
{[N]}\end{array}$ & \multicolumn{1}{c}{$\varepsilon_{\mathbf{r}}$} \\
\hline Anterior & aAC & 5000 & 0.06 \\
cruciate & pAC & 5000 & 0.10 \\
Posterior & aPC & 9000 & -0.24 \\
cruciate & pPC & 9000 & -0.03 \\
Lateral & aLC & 2000 & -0.25 \\
collateral & sLC & 2000 & -0.05 \\
& pLC & 2000 & 0.08 \\
Medial & aMC & 2750 & 0.04 \\
collateral & iMC & 2750 & 0.04 \\
& pMC & 2750 & 0.03 \\
Medial & aCM & 1000 & -0.18 \\
capsule & pCM & 1000 & -0.04 \\
\hline
\end{tabular}

simulated with the knee model. The differences of the internal-external rotations between the model and the experiments are minimized by variations of the reference strains of the line elements.

\section{Rigid articular contact}

For rigid articular surfaces, contact between femur and tibia will occur in contact points, of which the positions are a priori unknown and must follow from the equations describing the contact conditions. There are two contact conditions. Firstly, the points of contact $\mathbf{C}$ and $\mathrm{C}$ on the tibia and the femur must coincide yielding the contact position condition:

$$
\mathbf{c}=\mathbf{a}+\mathbf{R} \cdot \mathbf{c}
$$

where $\mathbf{c}$ and $\mathbf{R} \cdot \mathbf{C}$ are the position vectors of $\mathbf{C}$ and $C$, respectively. Secondly, the outward normal $\mathrm{n}$ at point $\mathbf{C}$ must have the opposite direction as the normal $\mathbf{R} \cdot \mathbf{A}$ at point $\mathrm{C}$, yielding the contact normal condition:

$$
\mathbf{n}=-\mathbf{R} \cdot \mathbf{A} \text {. }
$$

The normal $\mathbf{n}$ is calculated from

$$
n=s \frac{c_{v_{1}} \times c_{v_{2}}}{\left\|c_{v_{1}} \times c_{v_{2}}\right\|}, \quad s= \pm 1,
$$

where

$$
\mathbf{c}_{v_{1}}=\partial c / \partial v_{1}, \quad \cdot \mathbf{c}_{v_{2}}=\partial c / \partial v_{1},
$$

and $v_{1}$ and $v_{2}$ are orthogonal curvilinear coordinates of the surface and $s$ is chosen such that $a$ points outward. Note that $\mathbf{n}$ is a unit vector. The contact position condition and the contact normal condition form a set of $5 n$ equations, where $n$ is the number of contact points. The assumption of point contact implies that the curvatures of the surfaces at the points of contact on the femur and the tibia allow such contact to occur.

Since no friction is assumed in the articular contact, the contact force at contact point $\hat{C}_{k}$ is directed along the normal vector $\mathbf{R} \cdot \mathbf{f}_{\mathbf{k}}$. The magnitude of the contact force $p_{k}$ at contact point $k$ is introduced as an unknown parameter in the solution procedure. The total compressive contact force on the femur $f_{c}$ and contact moment $\mathbf{m}_{\mathrm{c}}$ about $\boldsymbol{O}$ are found by summation over $\boldsymbol{n}$ contact points:

$$
\begin{aligned}
\mathbf{f}_{\mathrm{c}} & =\sum_{1}^{n}-p_{\mathbf{k}} \mathbf{R} \cdot \mathbf{t}_{\mathrm{k}}, \\
\mathbf{m}_{\mathrm{c}} & =\sum_{1}^{n}-p_{\mathbf{k}} \mathbf{R} \cdot\left(\mathbf{k}_{\mathrm{k}} \times \hat{\mathbf{t}}_{\mathrm{k}}\right) .
\end{aligned}
$$

The contact force is regarded as being positive when contact actually occurs. If after solution of the equilibrium and contact equations a negative contact force is found, the contact will be eliminated and a new system of equations will be solved.

\section{Deformable articular contact}

In this study, the mathematical description of deformable articular contact is based on the simplified theory of contact from Kalker (1985) for thin layers of isotropic, linear-elastic material bonded to a rigid foundation. This implies that for the cartilage layer three assumptions are made. Firstly, the characteristic length of the contact area is assumed to be large relative to the cartilage thickness. Secondly, the cartilage layer is considered as an isotropic, linear-elastic material. This deviates from its more complex behaviour, which is described as non-linear, visco-elastic and biphasic (e.g. Mow et al., 1986; Mak, 1986; Kempson, 1980). However, the present knee model is quasi-static with no time-dependent characteristics. Deformable articular contact is used merely as a first order approximation of the behaviour of the articular contact. Thirdly, the subchondral bone is being considered as rigid. The simplified contact description is a first order approximation of the relation between the normal surface stress $\sigma_{n}$ and the surface displacement (Fig. 2):

$$
\sigma_{\mathrm{n}}=S\left(u_{\mathrm{n}} / b\right)
$$

with

$$
S=\frac{(1-v) E}{(1+v)(1-2 v)}
$$

where $\sigma_{\mathrm{m}}$ is the surface stress perpendicular to the surface, $u_{n}$ is the surface displacement, $b$ is the surfaces

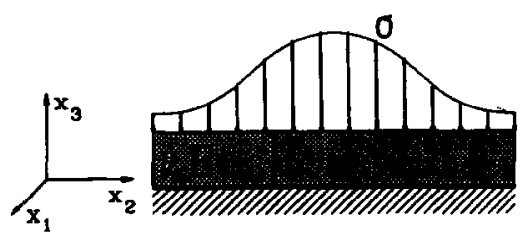

Fig. 2. The parameters involved in the simplified model for articular contact. The thickness $b$ of the soft layer, the surface stress perpendicular to the surface $\sigma$, and the elastic modulus $E$ and Poisson's ratio $v$ describing the linear elastic properties of the soft layer. 
thickness, $E$ is the elastic modulus and $v$ is the Poisson's ratio. This deformation model is equivalent to the articular surface model employed by An et al. (1990) in their two-dimensional analysis of pressure distribution in a joint. This description of articular contact deformation is strictly linear and will only be valid for small surface displacements. The stifiness parameter $S$ is the confined compression modulus or the aggregate modulus (Mow et al., 1982). For large surface displacements, geometrical non-linear behaviour can be accounted for by an integration over the total displacement of the incremental stress increase as a function of the incremental displacement. The incremental stress increase is given by

$$
\mathrm{d} \sigma_{\mathrm{n}}=S \frac{\mathrm{d} u_{\mathrm{a}}}{\left(b-u_{\mathrm{n}}\right)} .
$$

Division of the pumerator and the denominator by the thickness $b$, and subsequent integration over the relative surface displacement $u_{n} / b$ yields

$$
\sigma_{\mathrm{n}}=\int_{0}^{u_{\mathrm{n}} / b} S \frac{\mathrm{d}\left(u_{\mathrm{n}} / b\right)}{\left[1-\left(u_{\mathrm{a}} / b\right)\right]}=-S \ln \left[1-\left(u_{\mathrm{a}} / b\right)\right]
$$

This relation represents an effect similar to strain hardening, since the stiffness increases with increasing surface displacement:

$$
\mathrm{d} \sigma_{\mathrm{n}} / \mathrm{d} \varepsilon_{\mathrm{n}}=S\left(1-\varepsilon_{\mathrm{n}}\right), \text { with } \varepsilon_{\mathrm{n}}=u_{\mathrm{d}} / b \text {. }
$$

In the present model, where two bodies are in contact, the material properties of the cartilage of both bodies are assumed to be equal. The parameter $b$ in equation (15) is then equal to the total thickness of the two contacting layers. The surface displacement $u_{\mathrm{a}}$ is the sum of the relative tibial and femoral surface displacements, which is obtained by the surface penetration of the undeformed tibial and femoral surfaces (Fig 3).

The compressive contact force on the femur $f_{c}$ and contact moment $m_{c}$ about $\boldsymbol{O}$ are evaluated by integration of the contact stresses over the femoral surface $\Omega$ :

$$
\mathbf{f}_{c}=-\mathbf{R} \cdot \iint_{\Omega} \hat{\sigma}_{\mathrm{v}} \mathbf{A} \boldsymbol{\Omega}
$$

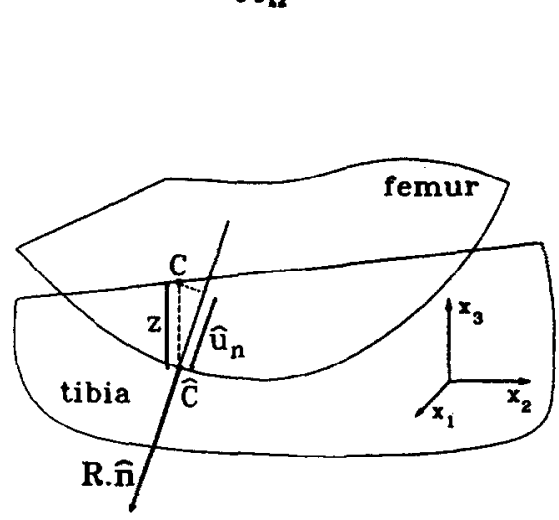

Fig. 3. The penetration model unod to determine the surfice displacement relative to the epaco-fixed coondinate syatem from two surface points $\mathbf{C}$ and $\mathbf{C}$ on the femur and tibia, respectively.

$$
m_{c}=-R \cdot \iint_{\Omega} \hat{\sigma}_{\mathrm{q}} \mathrm{e} \times \mathbf{d} \mathbf{d}
$$

with

$$
\begin{gathered}
\hat{\sigma}_{\mathrm{n}}=\hat{\sigma}_{\mathrm{n}}\left(\hat{u}_{\mathrm{n}}\right), \\
\hat{u}_{\mathrm{n}}=z e_{3} \cdot \mathbf{R} \cdot \mathbf{n}=\left[c_{3}-a_{3}-(\mathbf{R} \cdot \boldsymbol{C})_{3}\right] \mathrm{e}_{3} \cdot \mathbf{R} \cdot \mathrm{A}
\end{gathered}
$$

in which $z$ is the penetration of the femoral condyle into the tibial plateau (Fig 3), in the direction of $x_{3}$ (which is, of course, equal to the penetration of the tibial plateau into the (emoral condyle) and $e_{3}$ is the unit vector pointing in the direction of $x_{3}$.

The integrals (16a) and (16b) are numerically evaluated with the simplest integration rule, the composed midpoint rule (Davis and Rabinowitz, 1975). An $\boldsymbol{n}$ by $m$ grid is laid over the femoral condyles by using the spherical coordinates $f=f(a, f)$, where by the grid size is given by $(f \Delta \alpha, \boldsymbol{\Delta} \mu)$. The area of each spherical rectangle is given by (Fig. 4):

$$
A_{i j}=f_{i j}^{2} \cos \alpha_{i j} \Delta \alpha \Delta \beta \text {. }
$$

On each rectangle the displacement $a_{n}$, and thus the normal surface stress, is considered to have a constant value. This reduces the integration to a summation procedure of the products of area $A_{j}$ and midpoint stress $\delta_{n}$. Applying the integration rule to the integrals of equations (16) yields

$$
\begin{aligned}
\mathbf{f}_{c} & =-\mathbf{R} \cdot \sum_{j=1}^{m} \sum_{i=1}^{n}\left[\hat{\sigma}_{n} \Delta r_{i j}^{2} \cos \alpha_{i j} \Delta \alpha \Delta \beta\right], \\
m_{c} & =-\mathbf{R} \cdot \sum_{j=1}^{m} \sum_{i=1}^{n}\left[\hat{\sigma}_{n}(\hat{c} \times \hat{\theta}) r_{i j}^{2} \cos \alpha_{i j} \Delta \alpha \Delta \beta\right] .
\end{aligned}
$$

The description of deformable articular contact will thus only contribute to force and moment equations without the need of additional contact equations.

\section{Resulting model equations}

Equilibrium of the sum of the forces and the moments will give six equations:

$$
f_{0}+f_{p}+f_{1}+f_{c}=0
$$

and

$$
m_{6}+m_{r}+m_{1}+m_{c}=0
$$

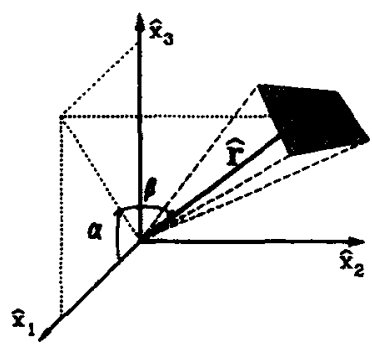

Fig. 4. The decaription of the curfece element as und in the numerical integration over the femoral articular surface. 
in which $f_{e}$ and $m_{e}$ are the externally applied forces and moments; $f_{r}$ and $m$, the constraint loads, equation (2); $f_{l}$ and $m_{l}$ the total ligament loads, equation (5); and $f_{c}$ and $m_{c}$ the contact loads either from the rigid contact description, equation (12), or the deformable contact description, equation (17). For each kinematic constraint the prescribed translation or rotation is eliminated from the equations and is substituted by an unknown constraint parameter $\sigma_{i}$, equation (2). For each rigid contact, five equations follow from the contact position condition, equation (9) and the contact normal condition, equation (10); and five unknown parameters are introduced, i.e. four parameters for the location of the contact point of the tibia and the femur, and one for the magnitude of the contact force. Hence, the total system is described by $(6+5 n)$ equations and an equal number of unknowns, in which $n$ is the number of rigid contact points. In the case of deformable contact, $n$ equals zero. The equations are solved by using a Newton-Raphson iteration procedure.

\section{Model analyses}

The geometry data of a knee specimen from the experimental studies of Blankevoort et al. $(1988,1990$, 1991) were used as input for the knee model. The locations of the ligament insertions were obtained by Roentgenstereophotogrammetry (Blankevoort et al., 1991). The geometry of the articular surfaces was measured by a stereophotogrammetric method from Meijer et al. (1989). The geometry data points were used to obtain the parameters for the surface polynomials by a least-squares fit (Wismans et al., 1980) of each of the four surfaces, i.e. the medial and lateral tibial plateaux and the medial and lateral femoral condyles. The simulated motions were two flexion motions similar to the motion pathways along the envelope of passive motion from Blankevoort et al. (1988). These were flexion motions with an axial torque of $3 \mathrm{Nm}$ applied around the $x_{3}$-axis, in internal rotation for the first motion pathway and in external rotation for the second motion pathway. When using rigid contact in the knee model, unpredictable results might arise if a contact point is eliminated for negative contact forces, because no new contact points can thereafter be generated. Therefore, no contact loosening was allowed. This was achieved by applying a compressive force of $-150 \mathrm{~N}$ on the femur which was directed parallel to the tibial $x_{3}$-axis.

The effect of the surface descriptions on the motion characteristics was studied by three parameter variations. The first gave a comparison between different polynomial approximations of the tibial surfaces: a close approximation by high degree polynomials, a less close approximation by low degree polynomials and a crude approximation by flat planes. Only the model simulations with the plane approximations of the tibial surfaces were performed for both the rigid and the deformable contact, since the rigid contact conditions could not be satisfied in all joint positions for the curved surface approximations. The femoral articular geometry was described in all cases by high degree polynomials using the spherical coordinates. A graphical representation of the different descriptions of the tibial surfaces is given in Fig. 5. The interpolation fit [Fig. 5(c)] was obtained through local linear interpolation of the data points around equally spaced grid points of a $x_{1} x_{2}$-grid. This resulted in a close match with the measured data points of the surfaces. The plane approximations [Fig. 5(d)] resulted from a least-squares fit of a plane through the data points of the medial and lateral surface. The plane approximations then gave the relative orientation of the surfaces. The curved surfaces as obtained from a least-squares fit of fourth degree polynomials to the data points showed the rough curvature patterns. The representation with the high degree polynomial fits of the surfaces, medial degree 6 and lateral

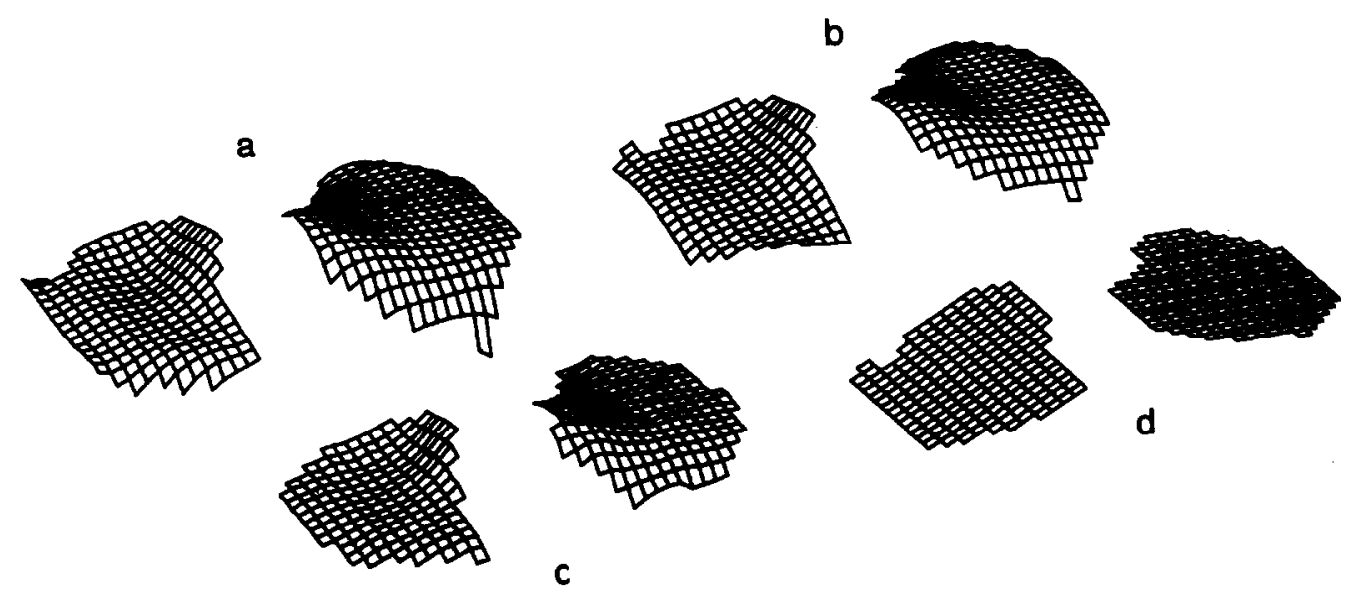

Fig. 5. Comparison of the different polynomial descriptions for the tibial surfaces with an interpolation fit to the geometry data points. (a) High degree polynomials, lateral degree 7 and medial degree 6; (b) low degree polynomials, lateral degree 4, medial degree 4; (c) interpolation fit; (d) planes. (The medial surface is on the left, the lateral surface is on the right) 


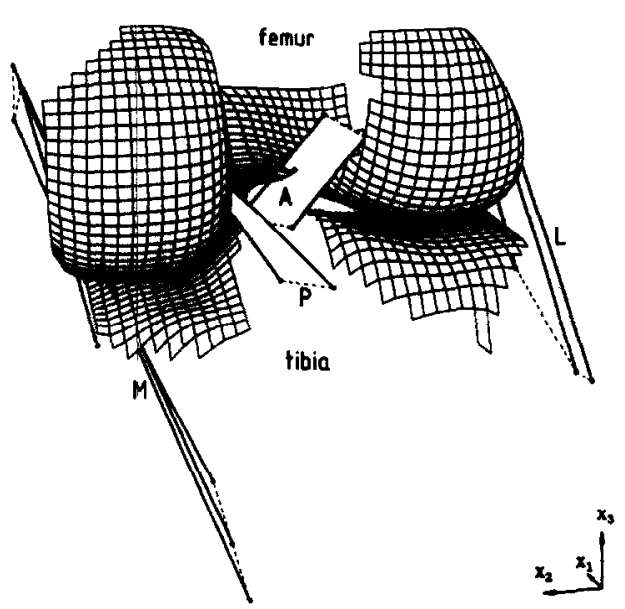

Fig. 6. Representation of the geometry of the knee model, including the femoral condyles, the tibial surfaces (high degree polynomialsh) and the line elements which model the four major ligaments (ACL, $\mathrm{PCL}, \mathrm{LCL}, \mathrm{MCL}$ ), for the joint in extension.

degree 7, gave a good description of the surfaces, as was indicated by the S.D. of 0.27 and $0.39 \mathrm{~mm}$ relative to the data points of the medial and lateral tibial surface, respectively. Figure 6 shows the geometry of the knee model for the parameter analysis with the high degree polynomial approximations of the tibial surfaces and the femoral condyles, and the line elements which represented the four ligaments.

The second parameter variation was a comparison between the linear and the non-linear deformable contact using the high degree polynomial approximations of the tibial surfaces. The third parameter variation was of the elastic modulus of the linear deformable surface description. Since normal articular cartilage shows a time-dependent response to mechanical loading, the elastic modulus and Poisson's ratio will have to be different for an instantaneous response and for a response after a long time-period. In the former case, the range of the elastic modulus is $5-15 \mathrm{MPa}$ with a Poisson's ratio close to 0.5 , whereas in the latter case, the elastic modulus is close to $1 \mathrm{MPa}$ with Poisson's ratio ranging from 0.0 to 0.4 (Mow et al., 1982; Kempson, 1980). The time span between successive positions in the experiments was in the order of $1 \mathrm{~min}$ (Blankevoort et al. 1988), which is considered to be neither a short time nor a long time, hence the choice was rather arbitrary. The elastic modulus was set at $5 \mathrm{MPa}$ and the Poisson's ratio at 0.45 . The thickness of the articular layer was assumed to have a constant value of $2 \mathrm{~mm}$ for all articular surfaces (Walker and Hajek, 1972; Roth, 1977).

\section{RESULTS}

The axial rotations and the varus-valgus rotations, as functions of flexion for the envelope of motion, are shown in Figs 7(a) and (b) for different surface descriptions and compared with the experimentally obtained
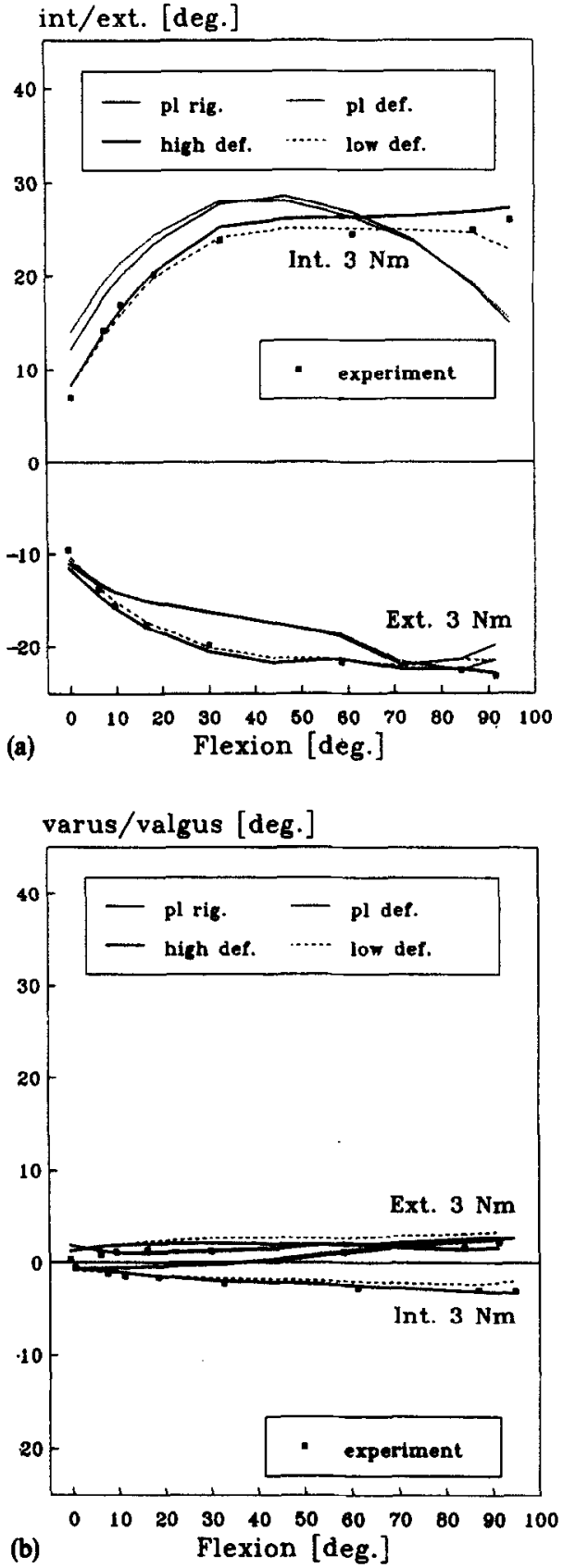

Fig. 7. Axial rotation (a) and varus-valgus rotation (b) as functions of flexion for the envelope of passive knee-joint motion as simulated by the knec models with difierent descriptions of the tibial surfaces: (pl rig.) rigid planes, (pl. def.) deformable planes, (low def.) deformable low degree polynomials and (high def.) deformable high degree polynomials.

values for this particular joint specimen. The anterior-posterior translation, medial-lateral translation and proximal-distal translation as functions of flexion are given in Figs 8(a) $-(f)$. The comparison between the plane approximations ( $\mathrm{pl}$. def.) and the curved polynomial approximations (high def.) for the tibial surfaces showed the largest differences. The 

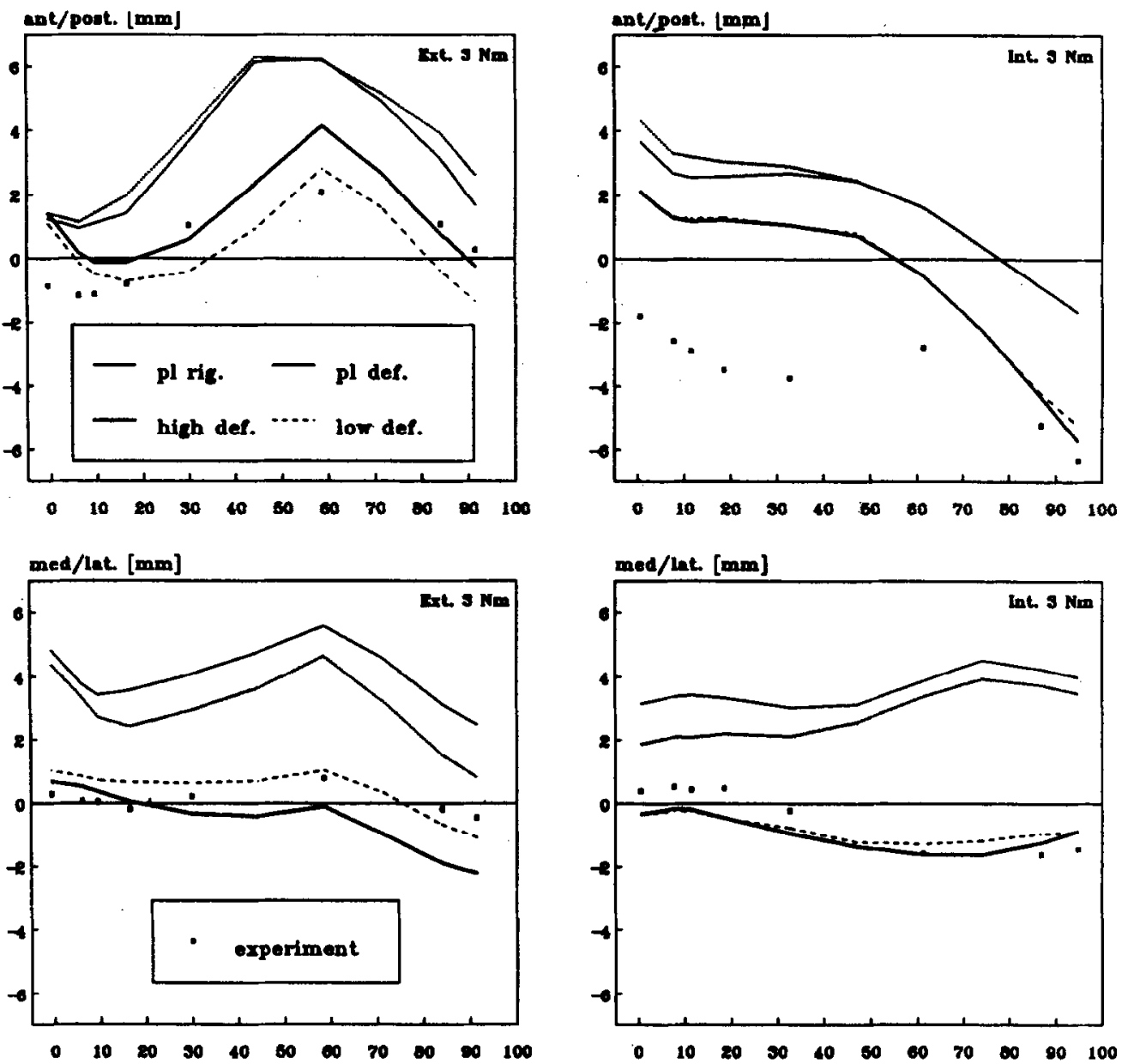

prox/ditht. [mm]
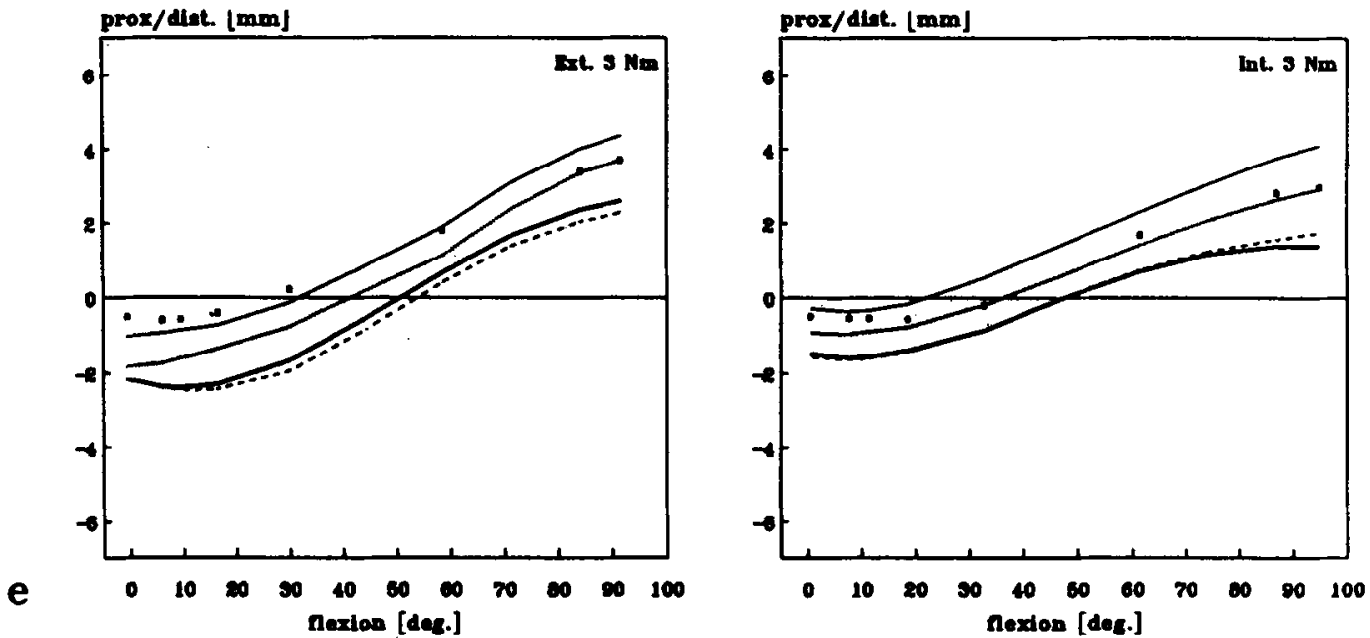

$\mathbf{f}$

Fig. 8. The translations of the femoral origin as functions of fexion for the envelope of paccive knoe-joint motion as simulated by the knee models with different descriptions of the tibial surfaces: (pl. rig) rigid planes. (pl def.) deformable planes, (low def.) deformable low degree polynomials and (high def.) deformable high degree polynomials.

internal rotations for the plane approximation were quite different from the experimentally measured values and those simulated with the curved surfaces. Two translation components were also influenced as indi- cated by a more anterior and medial position of the femur as compared to the curved surface descriptions. Of course, the plane approximations were expected to give different motion patterns, since the ligament 


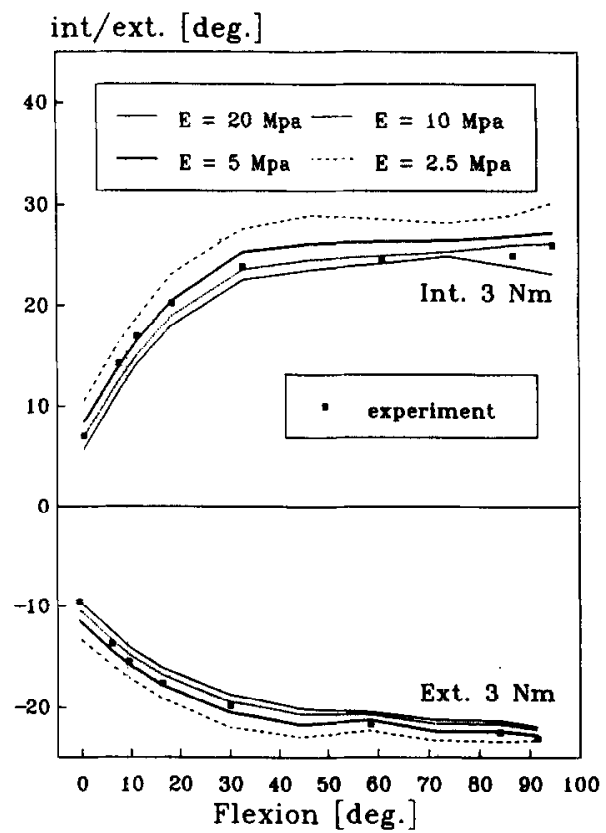

Fig. 9. The effect of variation of the elastic modulus of the linear deformable contact description on the envelope of passive knee-joint motion as simulated by the knee model with high degree polynomials for the tibial surfaces.

reference strains were adapted so as to match the model including the high degree polynomial approximations with the experimentally obtained internal and external rotations. The more medial position of the femur was probably caused by the lack of the medial concavity and the continuous medial slope in the flat plane approximation of the tibial surfaces. The difference between the rigid (pl. rig.) and deformable contact (pl. def.), as shown by the plane approximations, was small. Surprisingly, also the differnece between the low (low def.) and high (high def.) degree polynomial approximations was small, which means that probably only the rough curvature characteristics of the tibial surfaces were of importance for the motion characteristics.

Variation of the elastic modulus appeared to affect the axial rotations only (Fig. 9). With decreasing values, the surface deformations increased and as a consequence the ligaments relaxed a little, allowing more internal and external rotation to occur. Introduction of non-linear deformable contact in the knee model showed virtually no effect on the motion parameters relative to the model with linear deformable contact.

The contact surface displacements and the stress distributions on the femoral articular surface (Fig. 10) showed that the assumption of the characteristic length of the contact area being large relative to the cartilage thickness was a valid one. The maximal surface displacements and stresses occurred on the lateral condyle for internal rotation at $90^{\circ}$ flexion. These high contact stresses were caused mainly by the high tensions in the cruciate ligaments and for a much smaller part (approximately 10-15\%) by the axial force of $150 \mathrm{~N}$. The maximum resultant contact forces occurred at $95^{\circ}$ flexion for internal rotation and valued 620 and $440 \mathrm{~N}$ on the medial and lateral sides, respectively.

\section{DISCUSSION}

Mathematical models simulating the motion characteristics of human joints should incorporate a description for the articulating surfaces. The different approaches in mathematical knee models included rigid polynomial surfaces with point contact (Wismans et al., 1980; Wismans, 1980), hydrostatic finite elements distributed over possible areas of contact (Andriacchi et al., 1983) and deformable surfaces described by elastic compression springs at discrete data points on a surface (Essinger et al., 1989). The articular surface description introduced in this paper was based on a general theory for a thin elastic layer on a rigid foundation and can be applied in kinematic joint models in general. The advantage of deformable contact in the knee model over the rigid contact is that it is not restricted to contraform contact. Conforming surfaces can also be described by using the deformable contact; which means that the passive motion characteristics of almost any joint can be modelled. The second advantage concerns the numerical solution procedure. The mathematical description of the deformable contact contributes to the force and moment equations in the same way as the ligaments. Hence, no additional equations are necessary, as for rigid contact. Consequently, the knee model with deformable contact has a higher numerical stability (Kuiper, 1988).

The approximation of the articular surfaces by polynomials was the same as used by Wismans $e t$ al. (1980). In the case of rigid contact there was a limitation to the degree of the surface polynomials, since high local curvatures may result in contact areas where the conditions for point contact may not be satisfied for all joint positions. For deformable surfaces, however, there are no limitations to the curvatures and therefore the best fitting polynomial may be chosen to describe the articular surfaces. Other geometry descriptions may also be used, such as cubic spline patches (Scherrer and Hillberry, 1979; Huiskes et al., 1985). The model analyses showed that close approximations were not necessary, since the motion characteristics were not influenced greatly by the degree of the polynomial approximations for the curved tibial surfaces. This was caused by the size of the contact area (Fig. 10), which covered small surface irregularities and made the contribution of the surface stress distribution to the net contact force less dependent on the irregularities. For rigid contact, however, the surface irregularities would have had a large effect on the motion characteristics, since a small shift of the 

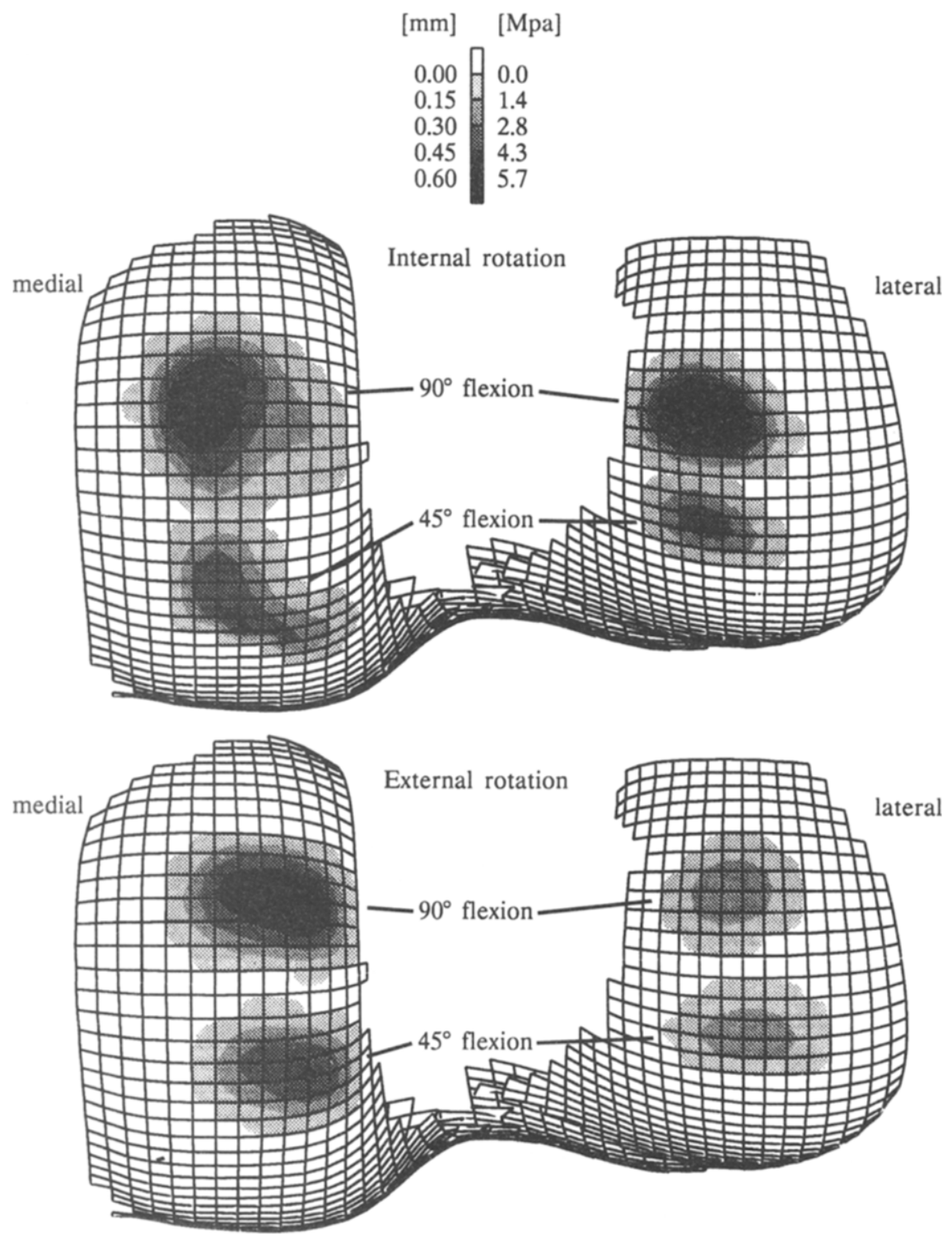

Fig. 10. Surface displacements [mm] and surface stresees [MPa] on the femoral articular surface at 45 and $90^{\circ}$ flexion, for external rotation and internal rotation with the linear deformable contact.

contact point over the surface would cause large direction changes of the surface normal at the contact point.

Deformable contact was merely introduced in the knee model to get round the problems arising from rigid contact if anatomically shaped articular surfaces are to be described. The exact nature of the local load transfer mechanism was of less importance. The timedependent characteristics of cartilage were not included in the model and, as a consequence, only a first order agreement may be expected with literature data in this respect, but only for data on menisectomized knees. The values of the surface stresses were in the range of those reported by Fukubayashi and Kurosawa (1980) and Ahmed and Burke (1983). In those studies the axially applied loads were much higher, ranging from 450 to $1800 \mathrm{~N}$, but the joints were not loaded to the limits of axial rotations as in the present study, where the ligament tensions appeared to be the main contributors to the contact stresses, which then reach values well in the range of the previously mentioned experimental studies.

The parameter variation showed that there is no need for a more precise assessment of the cartilage elastic modulus, since variations between 2.5 and $20 \mathrm{MPa}$ did not dramatically affect the passive motion characteristics. This, of course, holds only for using the model to study the passive motion characteristics for moderate loading conditions. Also, a more precise evaluation of the cartilage thickness in the case of linear deformation characteristics seems unnecessary since, according to equation (14), twice or half the 
thickness is similar to taking half or twice the elastic modulus, respectively. Introduction of non-linear deformable contact did not show to be of any importance when moderate loading conditions are assumed, as in the present study. The surface displacements at the contact area were too low as to expect any difference between linear and non-linear deformable contact, as was observed in the knee model while using an elastic modulus of $5 \mathrm{MPa}$. A lower elastic modulus, accounting for a longer time response, will certainly increase the strain hardening effect, but the contact surface area will increase as well. The relative contribution of the second order terms of the non-linear stress-strain relation, equation (15), will be limited to a small part of the contact area and its effect relative to the total contact area remains small. The linear deformable contact description, as a first order approximation of the non-linear descirption, may thus be used in the knee model when aiming at passive motion studies for moderate loading conditions. To study the contact mechanics or joint mechanics for high loading conditions may, of course, require nonlinear deformable contact, but a mathematical description of the menisci should then also be considered. It should be emphasized that the knee model can be used only within its intended scope, i.e. the simulation of the passive motion characteristics of the knee. For simulation of motion or loading conditions where the menisci play an important role, the simulation results of the meniscectomized model may be less or not reliable, as for instance the simulation of joint laxity in an ACL-deficient knee or motion studies with high axial loading.

The comparison between the model results and the experimental results for the envelope of passive motions showed a very good quantitative agreement with respect to all motion parameters, except for the APtranslations of the femoral origin for the internal motion pathway. From extension to $60^{\circ}$ flexion the femur is located too far anteriorly. This was caused by the uncertain nature of the choice of the ligament reference strains, which were adapted in such a way as to match the model simulations for internal and external rotations as functions of flexion with the experimentally obtained values. In this procedure, the pretensions of the anterior cruciate ligament bundles were probably taken too high. However, relaxing the ACL would increase the internal rotation drastically, which should then be compensated for by another ligament. This difficult point stresses the importance of finding a method to obtain values for reference strains in the ligaments, either by using the mathematical knee model or by some rigorous experimental method.

Acknowledgements-This research program was sponsored in part by Grant 90-90 from the Netherlands Organization for Research (NWO/MEDIGON). The basis of the computer programs of the knee model was developed by $L$. Dortmans at the Faculty of Mechanical Engineering of the Eindhoven University of Technology, The Netherlands. The authors appreciate the efforts of V. C. Mow and W. M. Lai from the New York Orthopaedic Hospital Rescarch Laboratory, Columbia University , New York, U.S.A. by reading critically the first manuscript and providing valuable comments and suggestions.

\section{REFERENCES}

Ahmed, A. M. and Burke, D. L. (1983) In vitro measurement of static pressure distribution in synovial joints-Part I: tibial surface of the knee. J. biomech. Engng 105, 216-224. An, K. N., Himeno, S., Tsumura, H., Kawai, T. and Chao, E. Y. S. (1990) Pressure distribution on articular surfaces: application to joint stability evaluation. $J$. Biomechanics 23, 1013-1020.

Andriacchi, T. P., Mikosa, R. P., Hampton, S. J. and Galante, J. O. (1983) Model studies of the stifiness chracteristics of the human knoe joint. J. Biomechanics 16, 23-29.

Bargar, W. L., Moreland, J. R., Markolf, K. L., Shoemaker S. C., Amstutz, H. C. and Grant, T. T. (1980) In vivo stability testing of post meniscectomy knees. Clin. Orthop. $150,247-252$.

Blankevoort, L. Huiskes, R. and Lange, A. de (1984) An in vitro study of the passive kinematic behavior of the human knee-joint. In 1984 Advances in Bloengineering (Edited by Spilker, R. L.). ASME, New York.

Blankevoort, L., Huiskes, R. and Lange, A. de (1988) The envelope of passive knee joint motion. J. Biomechanics 21, 705-720.

Blankevoort, L., Huiskes, R. and Lange, A. de (1990) Helical axes of passive knee joint motions. J. Biomechanics 23, 1219-1229.

Blankevoort, L., Huiskes, R. and Lange, A. de (1991) Recruitment of knee joint ligaments. J. biomech. Engng 113, 94-103.

Butler, D. L., Kay, M. D. and Stoufier, D. C. (1986) Comparison of material properties in fascicle-bone units from human patellar tendon and knee ligaments. J. Biomechancis 19, 425-432.

Crowninshield, R., Pope, M. H. and Johnson, R. J. (1976) An analytical model of the knoe. J. Biomechanics 9, 397-405.

Danylchuk, K. (1975) Studies on the morphometric and biomechanical characteristics of ligaments of the knee joint. M.Sc. thesis, Univeristy of Western Ontario, London, Ontario, Canada.

Davis, P. J. and Rabinowita, P. (1975) Methods of momerical integration. Academic Press, New York.

Essinger, J. R. Leyvraz, P. F., Heegard, J. H. and Robertson, D. D. (1989) A mathematical model for the evalustion of the behavior during flexion of condylar-type knee prostheses. J. Biomechanics 22, 1229-1241.

Fukubayashi, T. and Kurosaws, H. (1980) The contact area and pressure distribution pattern of the knee. Acta orthop. scand. 51, 871-879.

Girgis, F. G., Marshall, J. L. and AlMonajem, A. R. S. (1975) The cruciate ligaments of the knee joint. Clin. Orthop. 106, 216-231.

Grood, E. S. and Hefzy, M. S. (1982) An analytical technique for modelling knee joint stiffness-part I: ligamentous forces. J. biomech. Engng 104, 330-337.

Grood, E. S. and Suntay, W. J. (1983) A joint coordinate system for the clinical deacription of three-dimensional motions: application to the knee. J. biomech. Engng 105, 136-144.

Huiskes, R., Kremers, J., Lange, A. de., Woltring H. J., Selvik, G. and Rens, Th. J. G. van (1985) Analytical stereophotogrammetric determination of three-dimensional knee-joint geometry. J. Biomechanics 18, 559-570.

Kalker, J. J. (1985) A Course of Contact Mechanics. Delft University of Technology, Delft, The Netherlands.

Kempson, G. E. (1980) The mechanical properties of articular cartilage. In The Joints and Synovial Fluid, (Edited by 
Sokoloff, L.), Vol. II, pp. 177-238. Academic Press, New York.

Kuiper, J. H. (1988) Modeling of articular contact in a mathematical knee model. M.Sc. thesis, University of Twente, Enschede, The Netherlands.

Kurosawa, H., Walker, P. S., Abe, S., Garg, A. and Hunter, T. (1985) Geometry and motion of the knee for implant and orthotic design. J. Biomechanics 18, 487-499.

Mak, A. F. (1986) The apparent viscoelastic behavior of articular cartilage: the contributions from the intrinsic matrix viscoelasticity and interstitial fluid flow. J. biomech. Engng 108, 123-130.

Markolf, K. L., Bargar, W. L., Shoemaker, S. C. and Amstutz, H. C. (1981) The role of joint load in knee stability. J. Bone Jt Sturg. 63A, 570-585.

Meijer, R. C. M. B., Huiskes, R. and Kauer, J. M. G. (1989) A stereophotogrammetric method for measurements of ligament structure. J. Biomechanics 22, 177-184.

Mow, V. C., Kwan, M. K., Lai, W. M. and Holmes, M. H. (1986) A finite deformation theory for nonlinearly permeable soft hydrated biological tissues. In Frontiers in Biomechanics (Edited by Schmid-Schonbein, G. W., Woo, S. L. Y. and Zweifach, B. W.), pp. 153-179, Springer, New York.

Mow, V. C., Lai, W. M. and Holmes, M. H. (1982) Advanced theoretical and experimental techniques in cartilage research. In Biomechanics. Principles and Applications (Edited by Huiskes, R, van Campen, D. H. and de Wijn, J. R.), pp. 47-74, Martinus Nijhoff, The Hague.

Roth, V. (1977) Two problems in articular biomechanica: I. Finite element simulation for contact problems of articulations, II. Age dependent tensile properties. Ph.D. thesis, Rensselaer Polytechnic Institute, Troy, New York.

Scherrer, P. K. and Hillberry, B. M. (1979) Piecewise mathematical representation of articular surfaces. J. Biomechanics 12, 301-311.

Trent, P. S., Walker, P. S. and Wolf, B. (1976) Ligament length patterns, strength, and rotational axes of the knee joint. Clin. Orthop. 117, 263-270.

Walker, P. S. and Hajek, J. V. (1972) The load-bearing area in the knee joint. J. Biomechanics 5, 581-589.

Wismans, J. (1980) A three-dimensional mathematical model of the human knee joint. Ph.D. thesis, Eindhoven University of Technology, Eindhoven, The Netherlands.

Wismans, J., Veldpaus, F, Janssen, J, Huson, A and Struben, P. (1980) A throe-dimensional mathematical model of the knee-joint $J$. Biomechanics 13, 677-685.

Woltring, H. J. (1991) Representation and calculation of 3-D joint movement. Hum. mumt. Sci. 10,603-616.

\section{APPENDIX A}

Variation of the kinematic equation

For the evaluation of the kinematic constraints, the kinematic constraint vectors are obtained through the derivatives of the translation vector and the rotation matrix with respect to the translation and the rotation components, respectively. This is performed through variational calculus applied to the kinematic equation (1):

$$
\delta \mathbf{p}=\delta \mathbf{a}+\delta \mathbf{R} \cdot \mathbf{p} .
$$

This can be rewritten as

$$
\delta \mathbf{p}=\delta \mathbf{a}+\delta \mathbf{R} \cdot \mathbf{R} \cdot \mathbf{R} \cdot \mathbf{p}
$$

The rotation matrix $\mathbf{R}$ is proper orthogonal, so $\delta \mathbf{R} \cdot \mathbf{R}^{\mathbf{t}}$ is skew symmetric. A vector $\delta x$ exists such that for every $w$,

$$
\delta \mathbf{R} \cdot \mathbf{R}^{\mathbf{t}} \cdot \boldsymbol{w}=\boldsymbol{\delta} \boldsymbol{\pi} \times \boldsymbol{w} .
$$

Vector $\delta \pi$ is called the axial vector of $\delta \mathbf{R} \cdot \mathbf{R}^{t}$. Together with $\mathbf{R}$, this vector determines $\delta \mathbf{R}$ uniquely. The variation of the kinematic equation can be written as:

$$
\delta \mathbf{p}=\delta \mathbf{a}+\delta \boldsymbol{\delta} \times(\mathbf{R} \cdot \mathbf{p}) .
$$

One or more of the position parameters may be prescribed. These kinematical constraints can be written as:

$$
k_{1}(\mathbf{R}, \mathbf{R})=0 \text {. }
$$

The variation of this expression yields:

$$
k_{i}(\mathbf{a}+\delta \mathbf{n}, \mathbf{R}+\delta \mathbf{R})=0 \text {. }
$$

Using a Taylor-series expansion it can be shown that $\delta a$ and $\delta R$ are kinematically admissable, i.e. they do not violate the constraints if:

$$
a_{i} \cdot \delta \mathbf{n}+\boldsymbol{\beta}_{i} \cdot \delta \boldsymbol{z}=0
$$

Hence, the vectors $a_{1}$ and $\boldsymbol{\rho}_{1}$ are determined uniquely by the kinematical conditions.

\section{APPENDIX B}

\section{Ligament stiffness values}

The stiffness values for the line elements in the linear part of the force-strain relationship, as used by Wismans (1980), were based on data from Trent et al. (1976). Andriacchi et al. (1983) based his stifiness data also on Trent et al. (1976) but combined them with the data of Girgis et al. (1975). However, it is not clear how they obtained higher values as compared to Wismans (1980). In Wismans et al. (1980), the force-elongation relationship is quadratic:

$$
\begin{array}{ll}
F=k\left(L-L_{0}\right)^{2}, & L>L_{0}, \\
F=0, & L \leqslant L_{0} .
\end{array}
$$

This relation was also used by Essinger et al. (1989). Since this

\begin{tabular}{|c|c|c|c|c|}
\hline $\begin{array}{l}\text { Ligament } \\
\text { bundle }\end{array}$ & $\begin{array}{l}\text { Trent et al. } \\
\text { (1976) }\end{array}$ & $\begin{array}{l}\text { Wismans } \\
(1980)\end{array}$ & $\begin{array}{c}\text { Andriacchi et al. } \\
\text { (1983) }\end{array}$ & $\begin{array}{c}E \sim 345 \mathrm{~N} \mathrm{~mm}^{-2} \\
\text { Cross-scetional area } \\
\text { from Danylchuk (1975) }\end{array}$ \\
\hline $\begin{array}{l}\text { ACL } \\
\text { PCL } \\
\text { LCL } \\
\text { MCL } \\
\text { CM } \\
\text { CL }\end{array}$ & $\begin{array}{c}3041 \\
4483 \\
3051 \\
5160 \\
- \\
-\end{array}$ & $\begin{array}{l}3000 \\
4500 \\
3000 \\
8000 \\
1000 \\
1000\end{array}$ & $\begin{array}{r}7200 \\
14,300 \\
7300 \\
8200 \\
- \\
-\end{array}$ & $\begin{array}{c}12,075 \\
20,010 \\
- \\
8280 \\
- \\
-\end{array}$ \\
\hline
\end{tabular}
relation lacks a linear part, the stiffiness values can not be related readily to those of Wismans (1980) and Andriacchi et al. (1983). Butler et al. (1986) performed a study on the material properties of human knee ligaments by testing fascicle-bone units. An average value for the elastic modulus of $345 \mathrm{MPa}$ was observed for specimens from three relatively young donors (age 20-30 yr). Multiplication with the cross-

Table 2. Comparison of literature data for the ligament stiffness $k$ [N] 
sectional area will give the stiffness as Newton per unit strain. Using morphological data from Danylchuk (1975), the stiffness values are higher than in the above mentioned studies, in particular for the cruciate ligaments.

In Table 2 a comparison is given of the stiffness data, as Newton per unit strain, from different sources. The data from Andriacchi et al. (1983) were obtained by multiplication of their stifiness as Newton $\mathrm{cm}^{-1}$ with the length of the line element and summation over the line elements belonging to a ligament. The area data from Danylchuk (1975) were given only for the ACL $\left(35 \mathrm{~mm}^{2}\right)$, the PCL $\left(58 \mathrm{~mm}^{2}\right)$ and the MCL $\left(24 \mathrm{~mm}^{2}\right)$. In the present model analyses, the stifiness values of the ACL, PCL and MCL are based on a combination of the estimates of Andriacchi et al. (1983), the linear elastic modulus of Butler et al. (1986) and the cross-sectional area, from Danylchuk et al. (1975). The stiffness of the LCL is chosen from the model of Andriacchi et al. (1983) and that of the two bundles of the deep fibres of the MCL are mere estimates (Wismans, 1980). The stifiness of each ligament is divided equally over its line elements. 